\title{
Employer Branding: Was Kliniken von anderen Branchen lernen können
}

\author{
Anja Lüthy
}

Online publiziert: 26. Oktober 2012

(C) Springer-Verlag Wien 2012

Kliniken sind immer noch viel zu altmodisch. Mit den herkömmlichen Methoden der Personalsuche werden sie dem demographischen Wandel nicht erfolgreich begegnen können. Parallel zur Entwicklung einer mitarbeiterorientierten Unternehmenskultur sollten sie auch Social Media gezielt nutzen, um junges und gutes Personal zu finden.

Mitarbeiter der jüngeren Generation suchen ihre Stellen ausschließlich online, also müssen Krankenhäuser für einen ansprechenden Webauftritt sorgen, Online-Anzeigen auf einschlägigen Portalen schalten und sich bei Youtube und Facebook professionell und mitarbeiterorientiert präsentieren. Viele Häuser setzen bei der Personalsuche immer noch auf Headhunter und Leihfirmen, was sehr teuer und oft wenig erfolgreich ist.

Was können Kliniken in Sachen Personalmarketing von anderen Branchen lernen? Die Etablierung einer „gelebten" mitarbeiterorientierten Kultur, die sich sowohl intern bei den eigenen Mitarbeitern als auch nach extern herumspricht, ist erste Voraussetzung dafür, dass ein Unternehmen als attraktiver Arbeitgeber wahrgenommen wird. Dies kann dann - in einem zweiten Schritt - über gezieltes „Channelmanagement" kommuniziert werden: Alle Social-MediaAktivitäten werden systematisch so koordiniert, dass die Generation Y Lust bekommt, sich in dem entsprechenden Unternehmen zu bewerben.
Auf diese internetaffine Generation der 20- bis 25-Jährigen müssen sich auch Krankenhäuser unbedingt einstellen. Denn die Vertreter dieser Generation Y sind anspruchsvoll und selbstbewusst. Sie sind nicht mehr bereit, sich so zu knechten wie ihre Vorgängergenerationen. Sie wünschen sich wenig Überstunden, kompetente Führungskräfte, ständige Weiterentwicklung und eine ausgewogene Life-WorkBalance. Sie wollen einem Arbeitsplatz, an dem sie sich rundherum wohlfühlen.

In diesem Sinne sollten sich Krankenhäuser endlich auf den Weg machen, ihrem negativen Image entgegenzutreten und attraktive Arbeitgeber werden. Dabei hilft gezieltes Employer Branding, auch Arbeitgeber-Markenbildung genannt.

Andere Branchen haben längst erkannt, dass zu einem modernen Personalmanagement flexible und familienfreundliche Arbeitszeiten, motivierende Führungskräfte, Fort- und Weiterbildungsmöglichkeiten und nicht zuletzt ein gutes Arbeitsklima gehören. Nur wenn Mitarbeiter an ihren Arbeitsplätzen zufrieden sind, übernehmen sie gerne die Rolle von Marken-Botschaftern für ihr Unternehmen und ziehen damit neues Personal an.

A. Lüthy $(\bowtie)$

Berlin, Deutschland 